\title{
DENDROSOMAL CAPSAICIN NANOFORMULATION FOR THE INVITRO ANTI- CANCER EFFECT ON HEP 2 AND MCF -7 CELL LINES.
}

\author{
Carlin Geor Malar, Bavanilathamuthiah \\ Department of Biotechnology, Sathyabama University. \\ *carlinmalar@gmail.com
}

\begin{abstract}
-
In this study, the binding of the Capsaicin onto the dendrimers was investigated. The size, structure and morphology of the nanoparticles were determined using scanning electron microscopy (SEM) and X-ray diffraction (XRD) analysis. The binding of the Capsaicin onto the nano drug delivery carrier, dendrimer were initially confirmed by UV-Vis Spectrophotometer and further confirmed by Fourier transform infrared (FTIR) spectroscopy. The shape of dendrimers became distinct after the binding of capsaicin. The free dendrimers were found to be aggregated while DNCps were observed to exhibit good dispersability and defined shape because of the inert coating of the capsaicin. The average diameter of the dendrimer was found to be $96.2 \mathrm{~nm}$ while that of capsaicin bound dendrimers was $143.1 \mathrm{~nm}$. The maximum binding occurred at moderate capsaicin loading $(0.50 \mathrm{mg})$. The DNCps was found to show the anti-cancer effect against the MCF - 7 cells and the HEp 2 cells. The cytotoxicity of it against the cancer cells was at the concentration $0.62 \mu \mathrm{g} / \mathrm{mL}$, which was much lower than against the normal cells $(1.25 \mu \mathrm{g} / \mathrm{mL})$, hence enabling the administration of the nanoformulation against such cancer cells. By this study, it was concluded that DNCps can be used as an efficient drug against cancer.
\end{abstract}

Keywords: Dendrimers, capsaicin, nanoformulation, MCF - 7, HEp 2, anti-cancer.

\section{INTRODUCTION}

Cancer is a class of diseases that are characterized by cell growth in out of control. The condition becomes highly dangerous when it spreads to different parts of the body. Normally, cells in the body follow an orderly path of growth, division, and death but the cells that uncontrollably grow and do not die leads to the formation of cancer. This leads to a mass of abnormal cells that grows out of control. Cells can experience uncontrolled growth if there are damages or mutations to DNA, and therefore, damage to the genes involved in cell division. There is an association between the over expression of stem cell marker genes and progression of tumors. Hence targeting of self-renewal pathways can be used as a powerful approach in cancer treatment [4].

The use of nanotechnology in cancer treatment offers some exciting possibilities, including the possibility of destroying cancer tumors with minimal damage to healthy tissue as well as the detection and elimination of cancer cells before they form tumors. The use of precisely engineered materials to develop novel therapies and devices that may reduce toxicity as well as enhance the efficacy and delivery of treatments is the major application of nanotechnology to medicine. However, there are already several nanocarrier-based drugs on the market and much more nano-based therapeutics in clinical trials; the dendrimers are the efficient and highly preferred for targeting the cancer cells. Dendrimers are the nano-sized structures which consist of tree-like branches with many functional terminal ends. Dendrimers are also considered as the monodisperse macromolecules which contain symmetric branching units built around a small core molecule [3, 6 and 8]. These are only architectural structures and are not any compound [1]. Because of its polyvalent nature, it can exhibit multiple interactions with the target. One of the important properties of Dendrimers is its solubility which improves the bioavailability of the insoluble drugs and used as a powerful carrier for drug delivery.

One of the naturally occurring phytochemicals, Capsaicin which is the major pungent component of hot chilli peppers of the genus Capsicum (family Solanaceae) is used as a food additive. Capsaicin was found to have several physiological and pharmacological effects [2, 7]. Dendrosomal curcumin significantly decreased the relative expression of OCT4A, OCT4B1, SOX-2, and Nanog along with noticeable overexpression of miR-145 as the upstream regulator [5]. Curcumin is being explored 
much in the anti-cancer studies; capsaicin has been used in the present study.

\section{EXPERIMENTAL}

\section{Materials and Methods}

\section{A. Chemicals}

Oleoyl chloride, capsaicin and coomassie brilliant blue G 250 was purchased from Sigma-Aldrich (Bangalore, India). Polyethylene glycol 400 (PEG 400), chloroform, acetone, methanol and dimethyl sulphoxide and triethylamine were the products from Merck (Mumbai, India). Bovine serum albumin was purchased from $\mathrm{CDH}$ Laboratory Chemicals (New Delhi, India). Dulbecco's modified Eagle's medium (DMEM) and Roswell Park Memorial Institute medium (RPMI) were the product of Himedia (Mumbai, India). The Cell lines (VERO, HEp 2 and MCF - 7) were obtained from the Madras Veterinary College (Chennai, India). All the chemicals used were of analytical grade and Millipore water was used for all the experimental studies.

\section{B. Synthesis of Dendrimer}

The esterification process was used for the synthesis of the dendrimer [5]. The oleoyl chloride (0.01 $\mathrm{mol})$, polyethylene glycol $400(0.01 \mathrm{~mol})$ and triethylamine $(0.012 \mathrm{~mol})$ was added to the chloroform. The esterification process was allowed to take place for 4 hours at $25^{\circ} \mathrm{C}$. The organic phase was removed and the dendrimers were collected, dried under vacuum until completely dried.

\section{Synthesis of capsaicin bound dendrimer}

Various concentrations of capsaicin taken in acetone were added to the same amount of dendrimer (100 mg). The encapsulation efficiency was determined in order to optimize the amount of capsaicin to be added to the dendrimers. The successfully capsaicin bound dendrimers were recovered using filtration with acetone and was dried.

\section{Characterization}

The size, structure and morphology of the nanoparticles were determined using scanning electron microscopy (SEM - TESCAN VEGA3 SBU) and X-ray diffraction (XRD) analysis. The binding of capsaicin onto the dendrimers were initially known by the UV-Visible spectroscopy (Beckman). The formation of dendrimer and successful binding of capsaicin was confirmed by Fourier transform infra red (FT-IR) spectroscopy (Perkin Elmer Spectrum RX 1) using the potassium bromide $(\mathrm{KBr})$ pellet method in the range of $4000-400 \mathrm{~cm}^{-1}$.

\section{E. Anti-Oxidant Assay}

Equal volume of the nanoformulation suspension in the media was added to the T25 flasks with the VERO, HEp 2 and MCF - 7 cell lines. It was then incubated at 37 ${ }^{\circ} \mathrm{C}$ in $\mathrm{CO}_{2}$ incubator. On the following day, it was trypsinated and centrifuged to collect the cell pellet. The homogenized cells were resuspended in $100 \mu \mathrm{L}$ of the media and were used to estimate the reduced glutathione level and DPPH scavenging activity. The untreated cells were taken as control.

\section{F. Cytotoxicity assay}

In order to study the antitumor activity of dendrimers, capsaicin and dendrosomal capsaicin, it is important to determine the cytotoxicity concentration. Cultures were viewed using an inverted microscope to assess the degree of confluency and the absence of bacterial and fungal contaminants was confirmed. Cell monolayer was washed with PBS and Trypsin/EDTA was added on to the washed cell monolayer. The cells were examined using an inverted microscope to ensure that all the cells were detached and floated. $5 \mathrm{ml}$ of complete media was added and cells were triturated to get the cells in the form of suspension. MTT assay was performed to identify the cytotoxicity concentration of the nanoformulation.

\section{RESULTS AND DISCUSSIONS}

\section{A. UV-Vis Spectroscopy}

The absorbance peaks were recorded using the UVVisible Spectroscopy. The absorbance peak was found to be at $262 \mathrm{~nm}$ for the naked dendrimers. Capsaicin showed the characteristic peak at $278 \mathrm{~nm}$. There was a shift in the peak from $262 \mathrm{~nm}$ to $268 \mathrm{~nm}$ for the capsaicin bound dendrimers confirming the successful binding of capsaicin onto the dendrimer.

\section{B. $\mathrm{FT}-\mathrm{IR}$ analysis}

Figure 1 (a), (b) and (c) shows the fourier transform infrared spectrum of the dendrimer, DNCps and capsaicin 
respectively. The peaks confirmed the presence of the characteristic functional groups present in the dendrimer and binding of capsaicin. The presence of aliphatic $\mathrm{C}-\mathrm{H}$ (stretching band) was observed by the characteristic peak at $2929.8 \mathrm{~cm}^{-1}$. The $\mathrm{C}-\mathrm{H}$ bending vibration of $\mathrm{CH} 2$ can be seen at $1474 \mathrm{~cm}^{-1}$. The stretching vibration of $\mathrm{C}=\mathrm{C}$ was seen at $1652.8 \mathrm{~cm}^{-1}$. The stretching band of carbonyl group of ester ( $\mathrm{C}=\mathrm{O}$ and $\mathrm{C}-\mathrm{O}$ ) was found at $1720.3 \mathrm{~cm}^{-1}$ and $1104.2 \mathrm{~cm}^{-1}$ respectively.

The characteristic absorption bands of capsaicin were found at 3447.3, 2927.4, 2369.5, 1637.7, 1403.0, 1067.2 and $806 \mathrm{~cm}^{-1}$. The shifting of peaks were seen in the DNCps at 2939.3, 2358.3, 1638.4, 1474.7, 1067.9 $\mathrm{cm}^{-1}$. The appearance of peak in spectra of DNCps at $804.3 \mathrm{~cm}^{-1}$ confirmed that the capsaicin was bound to the dendrimer.

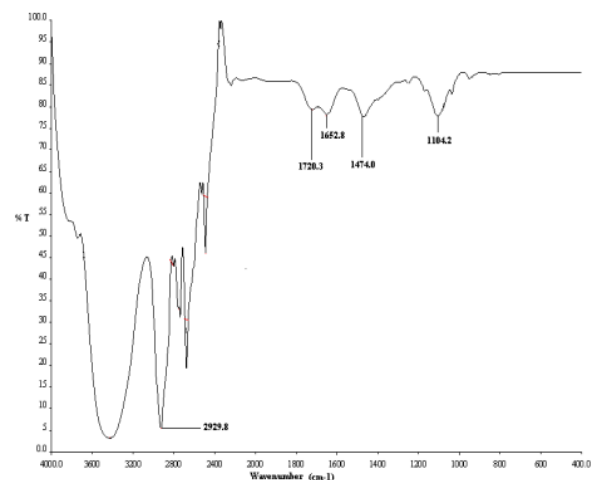

FT-IR Spectrum of (a) Dendrimer

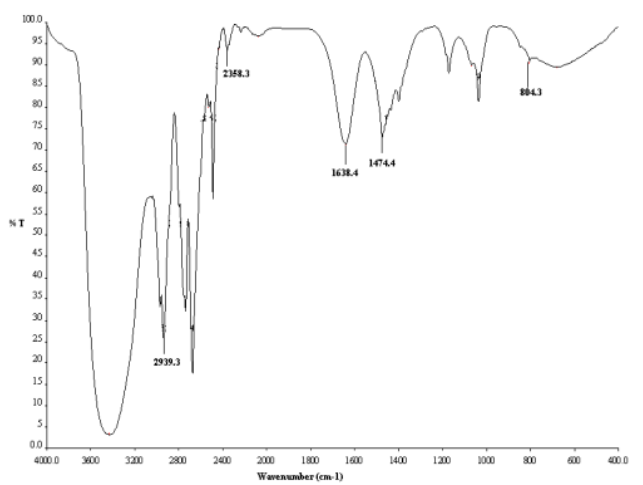

FT-IR Spectrum of (b) DNCps

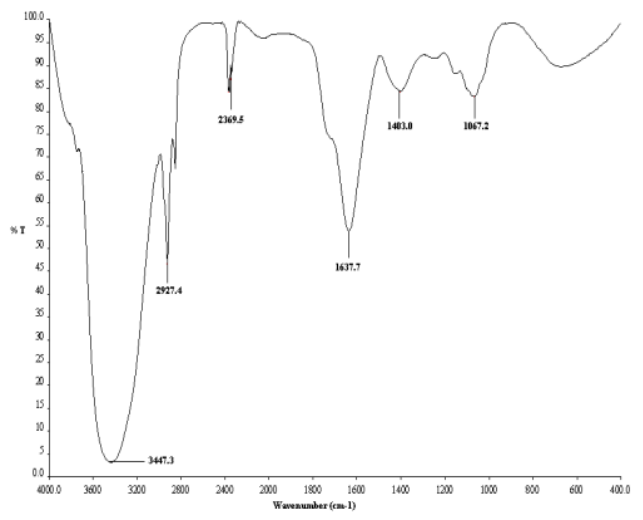

FT-IR Spectrum of (c) Capsaicin

Fig. 1 FT-IR Spectrum of (a) Dendrimer (b) DNCps (c) Capsaicin

\section{XRD analysis}

Fig.2 (a), (b) and (c) shows the XRD patterns of the naked and Capsaicin bound Dendrimers and Capsaicin respectively. There are series of characteristic peaks occurred at $2 \Theta$ for all the nanoparticles. The most intense characteristic peak was found to be at $28.16^{\circ}$ and $21.39^{\circ}$ for dendrimers and the DNCps respectively. With the XRD pattern, the average size of the particles can be calculated using the Debye-Scherrer formula $(D=K \lambda / \beta \cos \Theta)$ [Yan $H$ et al., 2009]. From the most intense peak with the corresponding FWHM, the average size of the particles was calculated to be $96.2 \mathrm{~nm}$ and $143.1 \mathrm{~nm}$ for dendrimers and the DNCps respectively.

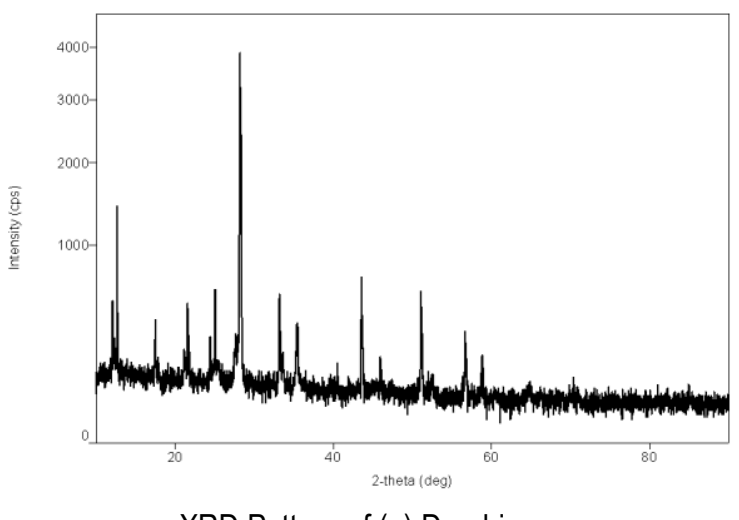

XRD Pattern of (a) Dendrimer 

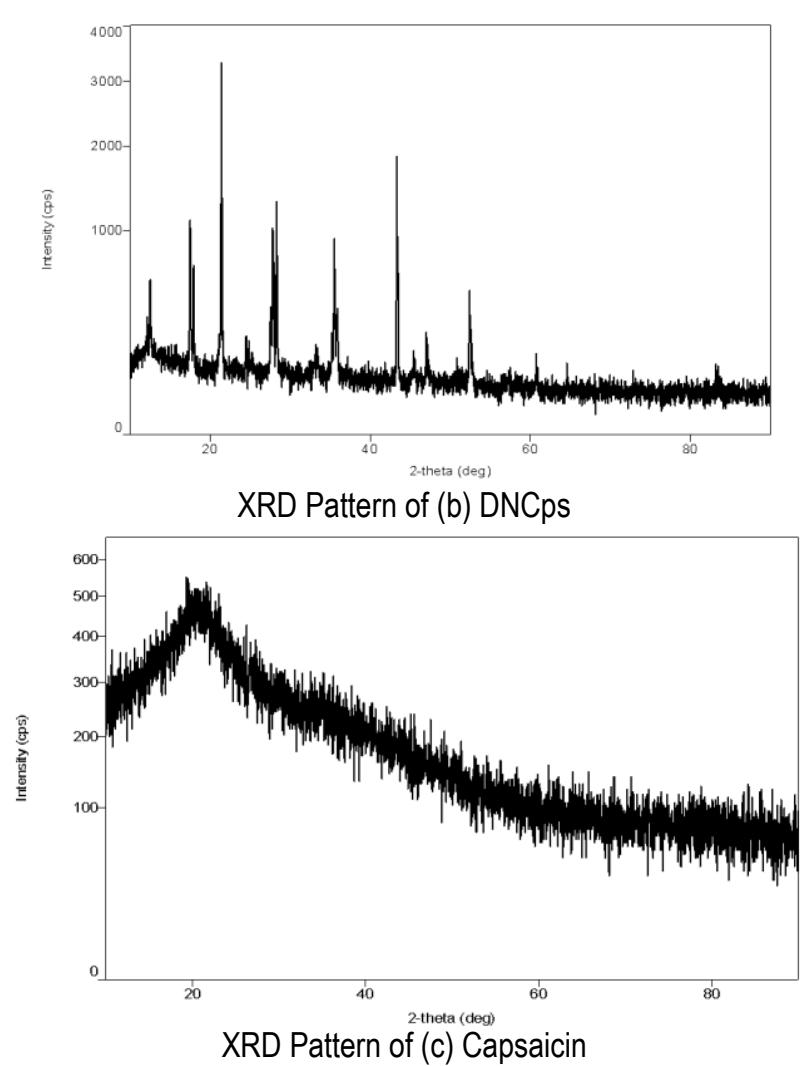

Fig. 2 XRD Pattern of (a) Dendrimer (b) DNCps (c) Capsaicin

\section{SEM analysis}

The shape and surface morphology of the dendrimers and capsaicin-loaded dendrimers were determined by the scanning electron microscopy. The free dendrimers were found to be aggregated which may be because of the highly reactive hydrophilic terminals while DNCps were observed to exhibit good dispersability and defined shape because of the inert coating of the capsaicin. The fig.3 (a) and (b) shows the SEM images of free dendrimers and capsaicin-loaded dendrimers respectively.

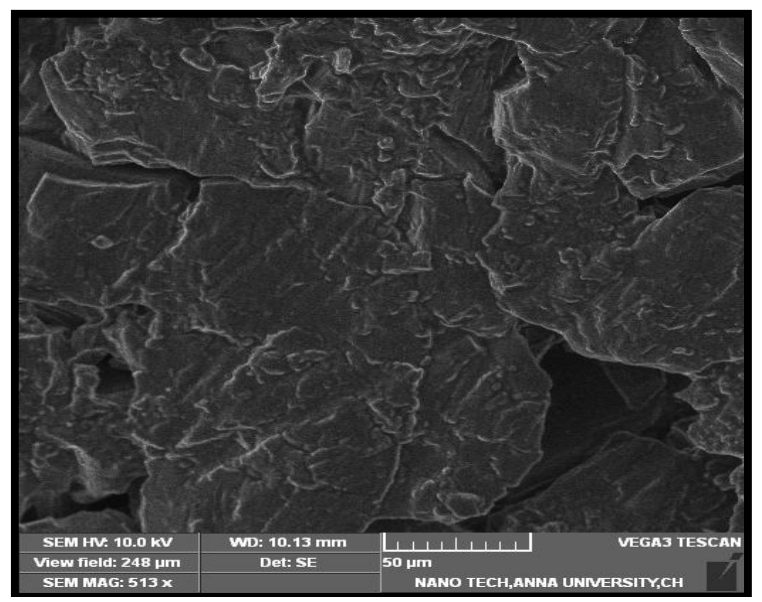

(a) Dendrimer

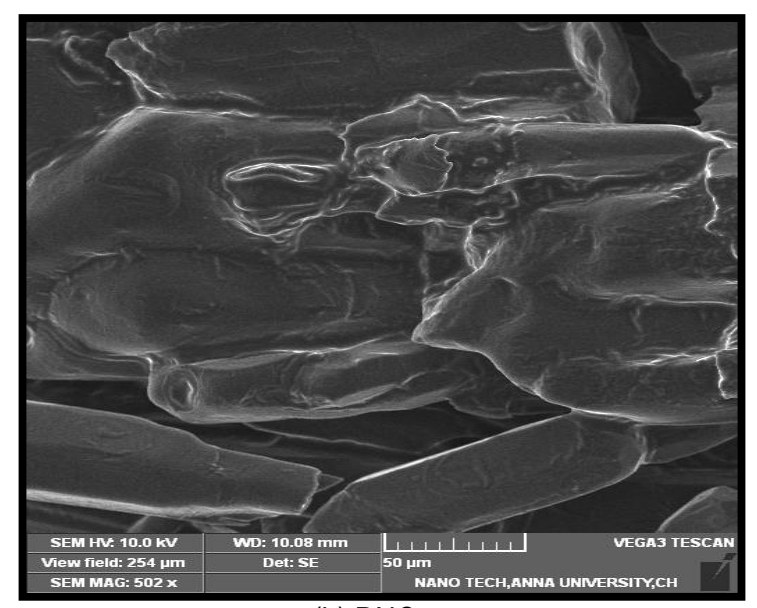

(b) DNCps

Fig.3. SEM image of (a) Dendrimer and (b) DNCps

\section{E. Anti-Oxidant activity}

\section{i. Estimation of Reduced Glutathione}

The concentration of reduced glutathione in the untreated MCF - 7 cells and HEp 2 cells was found to be less. The decreased GSH level may be due to higher requirement of GSH to carry out several processes involved in survival of cancer cells. There was little increase in the reduced glutathione level when the cancer cells were treated with dendrimers and capsaicin, whereas there was a notable increase in the reduced glutathione level when the cancer cells were treated with the nanoformulation, DNCps (fig.4 (a)). This shows the combined action of dendrimers and capsaicin was present in the DNCps. Hence the DNCps possess very good anti-oxidant property and can reduce the free radicals, one of the factors in cancer.

\section{ii. Estimation of DPPH Scavenging Activity}

The DPPH free radical scavenging activity was found to be less in the untreated cells. The reduction capability of the DPPH radical was determined by the decrease in its absorbance at $517 \mathrm{~nm}$, induced by antioxidants. The cells which were treated with the nanoformulation, DNCps showed the highest scavenging activity on both the cancer cells, followed by that of capsaicin and dendrimers. It was clear that the drug delivery carrier dendrimers also possessed the antioxidant activity, which is an additional advantage of using dendrimers. The DPPH scavenging activity is depicted in fig.4(b) 


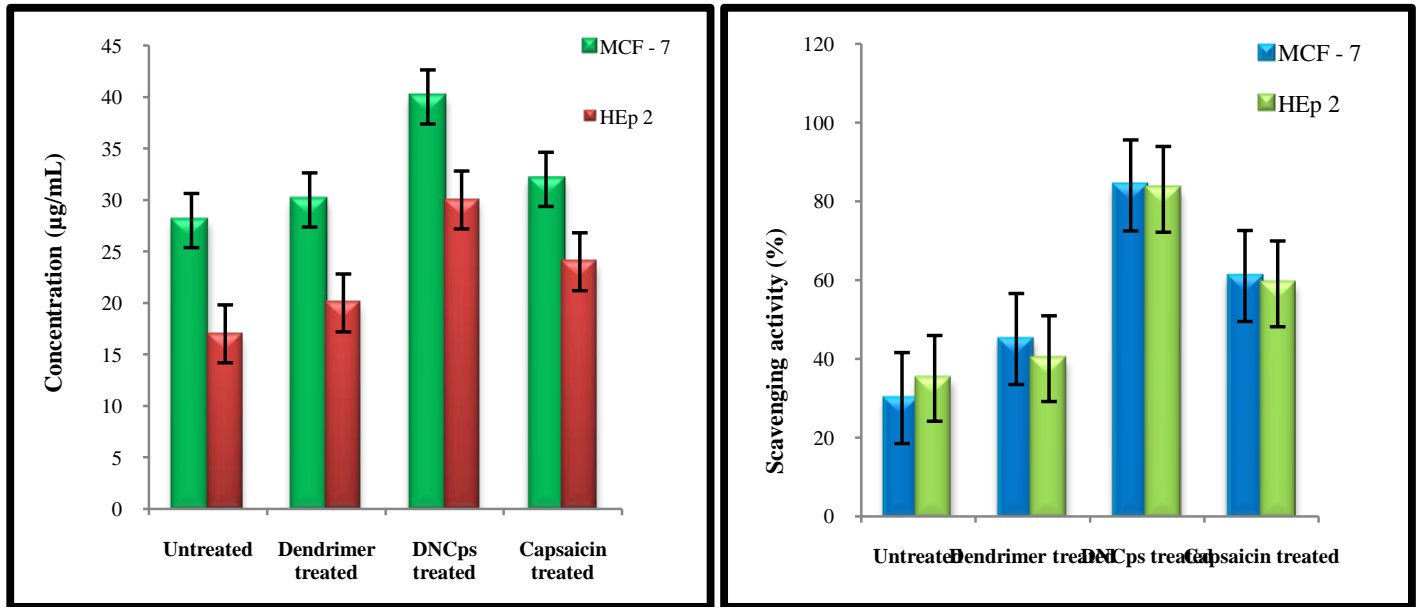

Fig.4. Effect of DNCps on (a) reduced glutathione level (b) DPPH Scavenging activity

\section{F. $\quad$ Cytotoxicity of Synthesized DNCps}

Treating the cells with the capsaicin bound dendrimers; the cytotoxicity of VERO cell lines was having an $\mathrm{IC}_{50}$ value of $1.25 \mu \mathrm{g} / \mathrm{mL}$ whereas the cytotoxicity of MCF - 7 and HEp2 cell was showing an $\mathrm{IC}_{50}$ of $0.62 \mu \mathrm{g} / \mathrm{mL}$. This study says that when the treatment with capsaicin bound dendrimers can cause cytotoxic effect for MCF - 7 cells and HEp2 cells at 0.62 $\mu \mathrm{g} / \mathrm{mL}$, where the same concentration would not affect the VERO cells (Fig.5, Fig. 6).

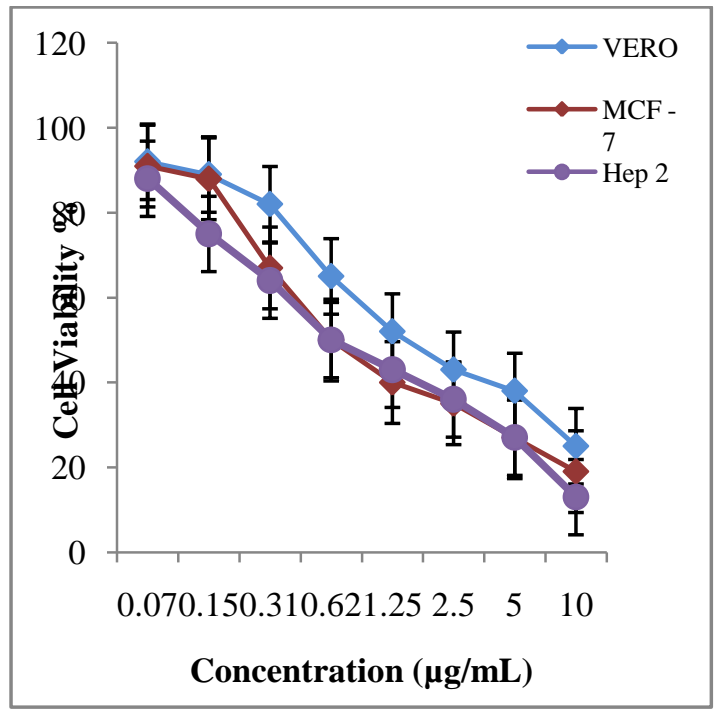

Fig. 5 Cytotoxicity when treated with DNCps

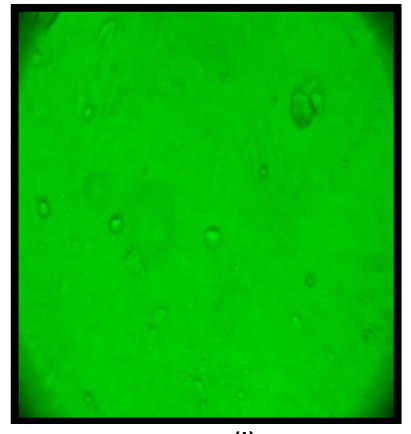

(i)

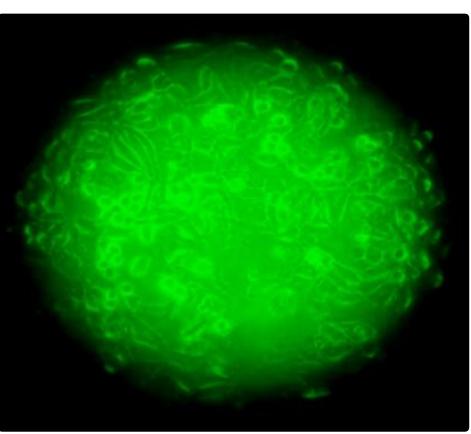

(iii)

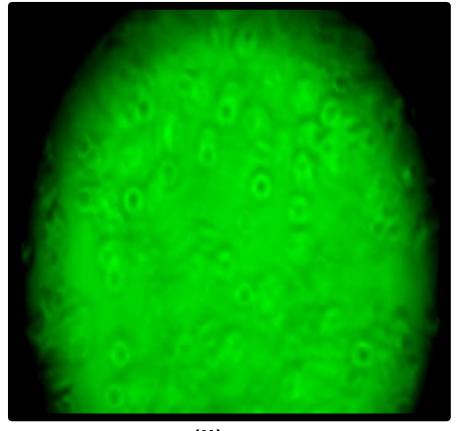

(ii)
Fig. 6 Cells treated with DNCps - VERO cells (i), MCF - 7 cells (ii) and HEp 2 cells (iii)

\section{CONCLUSION}

The dendrimers were synthesized by the esterification process and the capsaicin was coated onto it to prepare the nanoformulation. There was a shift in the peak from $262 \mathrm{~nm}$ to $268 \mathrm{~nm}$ for the DNCps. This shift was the preliminary confirmation of the successful binding of capsaicin onto the dendrimers. The synthesized dendrimers and DNCps were characterized using SEM. The FT-IR analysis showed the successful binding of 
capsaicin onto the dendrimers. The XRD analysis provided the size of the dendrimers was $96.2 \mathrm{~nm}$ which increased to $143.1 \mathrm{~nm}$ after the binding of capsaicin onto it. The synthesized DNCps showed the cytotoxicity on the normal and cancer cell lines at an $\mathrm{IC}_{50}$ value of 1.25 $\mu \mathrm{g} / \mathrm{mL}$ and $0.62 \mu \mathrm{g} / \mathrm{mL}$ respectively. Hence, the study says that the synthesized DNCps was effective against MCF -7 cells and HEp2 cells at $0.62 \mu \mathrm{g} / \mathrm{mL}$. At this concentration, they were not lethal to the normal VERO cells and hence can be used as an efficient drug nanoformulation against cancer.

\section{ACKNOWLEDGEMENTS}

We are very grateful to the Chancellor, the Directors of Sathyabama University for providing all necessary facilities and support to carry out this research in their well esteemed laboratory.

\section{REFERENCES}

[1] Abbasi E, Aval SF, Akbarzadeh A, Milani M, Nasrabadi HT, Joo SW, Hanifehpour Y, Nejati-Koshki K and Pashaei-Asl. Dendrimers: synthesis, applications and properties Nanoscale Research Letters, 9 (2014) 247.
[2] Govindarajan VS, Sathyanarayana MN: Handbook of Nutraceuticals and Functional Foods, Second Edition. Crit Rev Food Sci Nutr. 29: (1991) 435-474.

[3] Hawker CJ, Frechet JMJJ. Preparation of polymers with controlled molecular architecture. A new convergent approach to dendritic macromolecules. Am Chem Soc, 112 (21) : (1990) 7638-7647.

[4] Jordan CT. Cancer stem cell biology: from leukemia to solid tumors. Curr Opin Cell Biol.; 16 (6) : (2004) 708-712.

[5] Mirgani MT, Isacchi B, Sadeghizadeh M, Marra F, Bilia AR, Mowla SJ, Najafi F, Babaei E. Dendrosomal curcumin nanoformulation downregulates pluripotency genes via miR-145 activation in U87MG glioblastoma cells. Int $\mathrm{J}$ Nanomedicine: 9: (2014) 403-417.

[6] Newkome GR, Yao Z-Q, Baker GR, Gupta VK: Micelles. Part 1. Cascade molecules: a new approach to micelles. A [27]-arborol. J Org Chem, 50 (11): (1985) 2003-2004.

[7] Szallasi A, Blumberg PM: Vanilloid (Capsaicin) receptors and mechanisms. Pharmacol Rev, 51 (2): (1999)159-212.

[8] Tomalia DA, Baker H, Dewald J, Hall M, Kallos M, Martin S, Roeck J, Ryder J, Smith P: A new class of polymers: starburst-dendritic macromolecules. Polym J (Tokyo), 17: (1985) 117. 